\title{
openheart Patterns of ST segment resolution after guidewire passage and thrombus aspiration in primary percutaneous coronary intervention (PPCI) for acute myocardial infarction
}

\author{
Paul Russhard, ${ }^{1}$ Firas Al Janabi, ${ }^{1}$ Michael Parker, ${ }^{2}$ Gerald J Clesham ${ }^{1,2}$
}

To cite: Russhard $\mathrm{P}, \mathrm{Al}$ Janabi F, Parker M, et al. Patterns of ST segment resolution after guidewire passage and thrombus aspiration in primary percutaneous coronary intervention (PPCl) for acute myocardial infarction. Open Heart 2016;3:e000430. doi:10.1136/openhrt-2016000430

Received 8 March 2016 Revised 8 April 2016 Accepted 1 May 2016
${ }^{1}$ Department of Cardiology, Essex Cardiothoracic Centre, Nether Mayne, Basildon, Essex, UK

${ }^{2}$ Postgraduate Medical Institute, Anglia Ruskin University, Chelmsford, Essex, UK

Correspondence to Dr Gerald J Clesham; Gerald.Clesham@btuh.nhs.uk

\section{ABSTRACT}

Background: ST segment elevation allows the rapid identification of patients with acute myocardial infarction who benefit from emergency reperfusion. Primary percutaneous coronary intervention (PPCl) has emerged as the preferred perfusion strategy for patients presenting with ST segment elevation myocardial infarction (STEMI).

Methods and results: We studied the effects of the simple passage of an angioplasty guidewire followed by mechanical thrombus aspiration on the ST segment displacement in 289 patients presenting with acute STEMI. Simple guidewire passage led to a statistically significant fall in the mean ST elevation from 5.9 to $4.9 \mathrm{~mm}(p<0.001)$, but the mean ST displacement after subsequent mechanical thrombus aspiration was $4.8 \mathrm{~mm}$, not statistically significantly different from guidewire passage. When compared with simple guidewire passage, thrombus aspiration resulted in more patients achieving more than $50 \%$ ST resolution ( $21.8 \%$ vs $15.2 \%, p=0.009$ ), but a higher proportion had a worsening of ST elevation compared to baseline ( $19.7 \%$ vs $13.5 \%, p=0.041)$.

Conclusions: Mechanical thrombus aspiration in acute STEMI did not improve the mean ST resolution compared with simple guidewire passage. Thrombus aspiration increased the proportion achieving $50 \%$ resolution but also increased the proportion who had a worsening of ST elevation. These data may help explain some of the uncertainties surrounding the routine use of thrombus aspiration in STEMI and potentially supports the use of 'time to angioplasty guidewire passage' as one of the ways to judge the promptness of PPCI services.

\section{INTRODUCTION}

The finding of ST segment elevation in a patient presenting with chest pain allows rapid identification of those who benefit from emergency reperfusion. ${ }^{1}$ When patients were treated with thrombolytic agents, it was recognised that resolution of the ST segment

\section{KEY QUESTIONS}

What is already known about this subject?

- In acute ST segment elevation myocardial infarction (STEMI), primary percutaneous coronary intervention (PPCl) results in better resolution of ST elevation 90-120 min after treatment compared with thrombolysis. The role of routine thrombus aspiration in PPCl is uncertain.

What does this study add?

- Little data exist on ST segment resolution during $\mathrm{PPCl}$ procedures. We have described the degree of ST resolution after simple guidewire passage in PPCI. Thrombus aspiration does not increase the mean ST resolution compared with simple guidewire passage alone. We have demonstrated a mixed effect of thrombus aspiration on ST resolution with no net benefit.

How might this impact on clinical practice?

- On the basis of our data, 'time to guidewire passage' rather than 'time to device passage' could be used to assess the promptness of PPCI services. The mixed effect of thrombus aspiration on ST resolution may account for some of the uncertainties surrounding this procedure.

elevation yielded prognostic information. ${ }^{1}$ Indeed, rescue percutaneous coronary intervention emerged as a strategy to improve outcomes when there was failure of the ST segment elevation to resolve 90-120 min after the delivery of a thrombolytic agent. ${ }^{23}$

Primary percutaneous coronary intervention (PPCI) is now the preferred strategy for emergency reperfusion in patients presenting with ST elevation myocardial infarction (STEMI). It has been shown that PPCI is better than thrombolysis at achieving patency of the infarct-related artery. ${ }^{4}$ In addition, a large meta-analysis has suggested better 
outcomes for patients treated by PPCI. ${ }^{5}$ In clinical practice, there is less emphasis on the resolution of the ST elevation as a PPCI procedure usually restores flow in the epicardial coronary artery.

Despite the growth of PPCI for acute STEMI, the 30-day mortality figures for treated patients remain at around $6 \%{ }^{6}$ Prompt opening of the occluded artery needs to be accompanied by protection of microvascular flow. Poor distal flow in an unblocked artery, sometimes termed the no reflow phenomenon, is known to carry an adverse prognosis. ${ }^{7}$ Mechanical thrombus aspiration is widely used in PPCI with the aim of reducing distal embolisation although the outcome of this strategy, designed to protect microvascular flow, remains uncertain. 89

In this study, we evaluated ST segment elevation during PPCI procedures and, in particular, the immediate effects of guidewire passage and mechanical thrombus aspiration on ST resolution. In addition, we propose that the assessment of ST segment elevation during PPCI procedures may be used as a marker to evaluate strategies designed to protect the microvasculature of a patent infarct-related artery.

\section{METHODS}

We studied the ECG traces from 289 patients whose PPCI procedures were being audited at our institution. A standard Witt biomedical ECG system, used in our angiography laboratory, was used for all ECG recordings. A 12-lead trace was recorded at the start of the PPCI procedure immediately after the patient was connected up (ECG A). Furthermore, 12-lead ECG traces were recorded $30 \mathrm{~s}$ after passage of an angioplasty guidewire (ECG B) and $30 \mathrm{~s}$ after a thrombus aspiration catheter had been removed from the coronary artery (ECG C). A further trace was evaluated when the angioplasty operator deemed that the case was finished (ECG F).

All patients were loaded with aspirin $300 \mathrm{mg}$ and clopidogrel $600 \mathrm{mg}$ (given by the paramedics in the ambulance or in the emergency department). Unfractionated heparin $(70 \mathrm{U} / \mathrm{kg})$ was given once arterial access was obtained. The thrombus aspiration catheters used were the EXPORT (Medtronic) and the HUNTER (IHT Cordynamic). The use of adjunctive therapy (tirofiban) was at the discretion of the operator and typically given after thrombus aspiration had been performed.

\section{ECG analysis}

ST segment elevation was measured in the ECG lead with the maximum ST elevation on the initial recording. The ST elevation was measured $75 \mathrm{~ms}$ after the J point, and a proportion of the ECGs were independently assessed by a second individual to assess reproducibility of measurement. ECGs with left bundle branch block were excluded from this analysis.

\section{Statistical analysis}

A repeated measures of analysis of variance was used to compare the absolute ST segment elevation at different stages. Analysis was undertaken using the computer program R ( $\mathrm{R}$ Core Team. R: A language and environment for statistical computing. $\mathrm{R}$ Foundation for Statistical Computing, Vienna, Austria, 2015. http:// www.R-project.org). The repeated measures of analysis of variance was carried out as a linear mixed effects model with the calculations performed using the $\mathrm{R}$ package lme4 (D Bates, M Maechler, B Bolker, et al. lme4: Liner mixed-effects models using Eigen and S4. R package version 1.1-8, 2015. http://CRAN.R-project. org/package=lme4; D Bates, M Maechler, BM Bolker, et al, Fitting Liner Mixed-Effects Models using lme4. ArXiv e-print; Journal of Statistical Software, 2015. http:// arxiv.org/abs/1406.5823). The approximate F testing was obtained using the $\mathrm{R}$ package lmerTest (A Kuznetsova, P Brockhoff, R Christenses, lmerTest: Tests in Liner Mixed Effects Models, $\mathrm{R}$ package version 2.0-25, 2015. http://CRAN.R-project.org/package= lmerTest). The changes in the proportions of patients achieving 50\% reduction of ST segments and those demonstrating adverse ST segment changes were evaluated using McNemar's test.

\section{RESULTS}

We studied the ECGs from 289 patients presenting with STEMI. In all of these patients, guidewire passage was followed by mechanical thrombus aspiration before any other intervention, at the discretion of the operator. Of the patients, $94.8 \%$ later received a stent during the PPCI procedure. The mean age of the patients was 63 years, and $74.7 \%$ were men. In $47.4 \%$ of the patients, the lead with maximum ST elevation was in leads V1-V6, in $0.7 \%$ it was in leads I or aVl and in $51.9 \%$ the lead with maximum elevation was in leads II, III or aVf.

The mean initial ST segment elevation in the lead with maximum displacement was $5.9 \mathrm{~mm}$ (ECG A). The mean ST elevation after guidewire passage (ECG B) was 4.9 and $4.8 \mathrm{~mm}$ after thrombus aspiration (ECG C). The reduction of ST elevation after guidewire passage alone was statistically significant $(p<0.001)$, but the difference between guidewire passage and thrombus aspiration (ECG C compared to ECG B) was not statistically significant $(p<0.05)$. The mean ST elevation at the end of the case was $3.9 \mathrm{~mm}$ (ECG F), and this was statistically significantly lower than that after thrombus aspiration (ECG F vs ECG $\mathrm{C}, \mathrm{p}<0.001$ ). These data and CIs are shown in figure 1 .

A reduction of $50 \%$ or more of the initial ST elevation was seen in $15.2 \%$ patients after simple guidewire passage but was higher at $21.8 \%$ after thrombus aspiration $(p=0.009)$. At the end of the procedure, $44.3 \%$ of patients had a $50 \%$ or more reduction of ST elevation. Thus, thrombus aspiration does appear to increase the proportion of patients who achieve $50 \%$ resolution (figure 2).

In some patients, guidewire passage or thrombus aspiration increased the ST elevation seen on the initial 


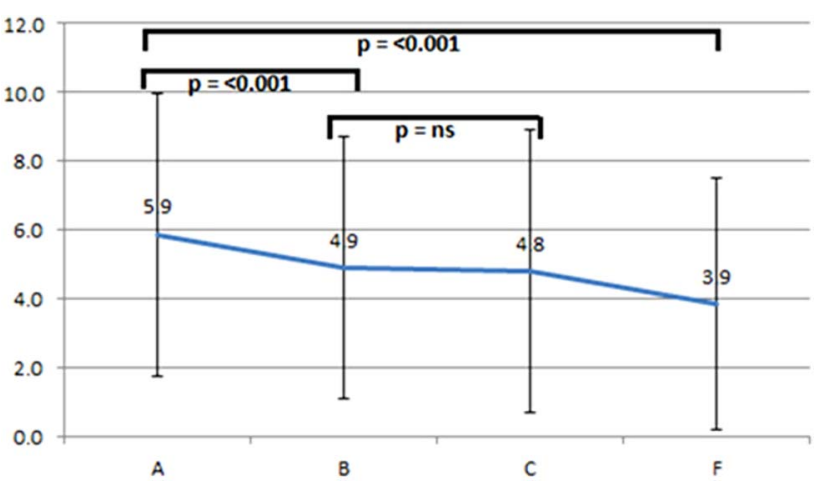

Figure 1 Absolute ST segment elevation at the beginning of the primary percutaneous coronary intervention procedure $(A)$, after guidewire passage $(B)$, after thrombus aspiration $(C)$ and at the end of the procedure $(F)$.

ECG (A). The proportion of patients with increased ST elevation compared to ECG A was $13.5 \%$ after guidewire passage and $19.7 \%$ after thrombus aspiration $(\mathrm{p}=0.041$, figure 3). In $12.5 \%$ of cases, the ST segment elevation was worse at the end of the procedure $(\mathrm{F})$. Although thrombus aspiration increased the proportion of patients achieving $50 \%$ resolution, it also increased the proportion who had a worsening of ST elevation.

\section{DISCUSSION}

Prompt reperfusion is recommended in acute myocardial infarction in order to reduce myocardial damage. ${ }^{10}$ Thrombolytic services were judged by how quickly the drug could be delivered after the arrival of the patient to hospital and the 'door to needle' time was widely used. For PPCI services, the equivalent measure is the 'door to balloon' time, ${ }^{11}$ which has now evolved to the 'door to device' time, as mechanical thrombus aspiration is widely used. Our data show that the mean ST resolution with thrombus aspiration is no greater than with angioplasty guidewire passage alone. There may be a case for using 'time to angioplasty guidewire passage' as

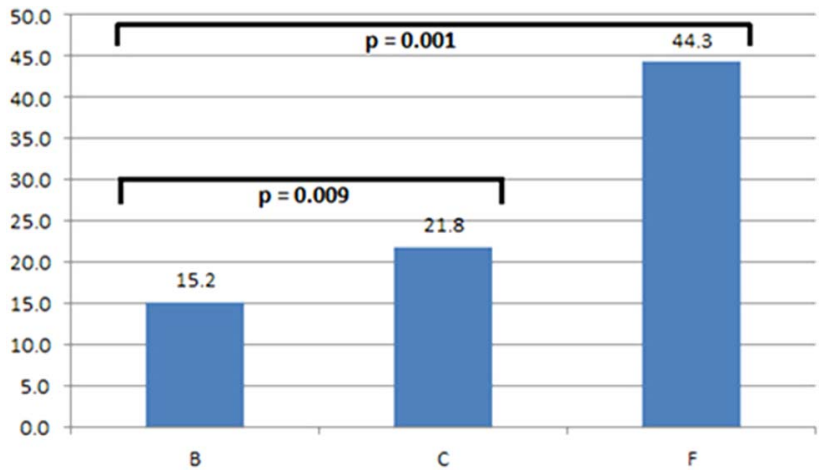

Figure 2 The percentage of patients who achieved more than $50 \%$ ST segment reduction after guidewire passage $(B)$, thrombus aspiration $(C)$ and at the end of the procedure $(F)$.

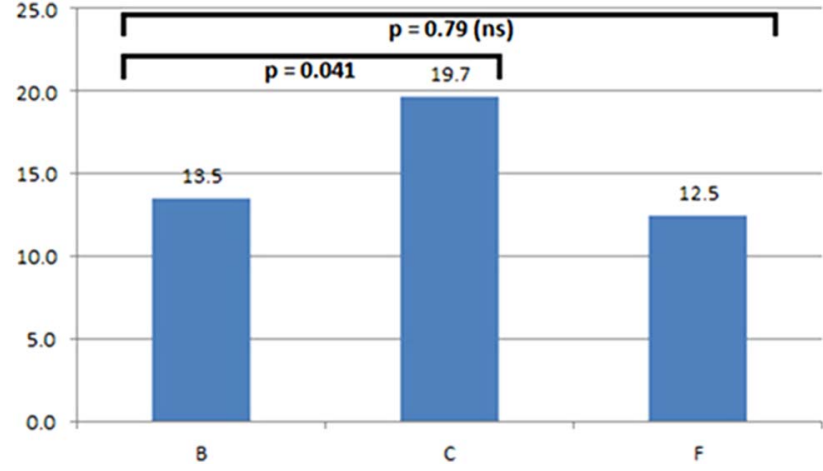

Figure 3 The percentage of patients where ST segment elevation is higher than at the start of the procedure. This is shown after guide passage (B), thrombus aspiration and at the end of the procedure $(\mathrm{F})$. $\mathrm{PPCl}$, primary percutaneous coronary intervention.

the measure to judge the promptness of a PPCI service once a patient arrives in hospital.

There appears to be a paradox in that thrombus aspiration contributes little to the mean ST resolution but increases the proportion of patients who do achieve more than $50 \%$ resolution. The explanation for this is that thrombus aspiration also increases ST elevation in some cases. It is possible that these relatively simple but bulky devices may be harmful in some cases possibly by causing embolisation of thrombus to the microcirculation.

Successful reperfusion in acute myocardial infarction involves opening the infarct-related artery and protecting the microcirculation. ${ }^{12}$ PPCI has been shown to be better than thrombolysis at achieving patency of the infarct-related artery and has become the preferred strategy for the treatment of acute STEMI. ${ }^{5}$ We have shown that mechanical thrombus aspiration can lead to ST resolution in some cases and worsening of the ST elevation in some cases. The overall effect of thrombus aspiration is made up of the net contribution of these two effects, and in our series thrombus aspiration was no better than guidewire passage alone in reducing ST elevation.

Our study shows that prompt ST resolution is seen in less than half of our series of patients undergoing PPCI for STEMI. We believe that other approaches to protect the microcirculation during PPCI may be beneficial and that careful monitoring of ST segment resolution during the procedure may be one of the ways of evaluating them.

Contributors GJC designed the project and wrote the manuscript. PR and FAJ analysed the ECGs, produced the figures and reviewed the manuscript. MP performed the statistical analyses.

Competing interests None declared.

Ethics approval Audit department, Essex Cardiothoracic Centre.

Provenance and peer review Not commissioned; externally peer reviewed. 
Data sharing statement No additional data are available.

Open Access This is an Open Access article distributed in accordance with the Creative Commons Attribution Non Commercial (CC BY-NC 4.0) license, which permits others to distribute, remix, adapt, build upon this work noncommercially, and license their derivative works on different terms, provided the original work is properly cited and the use is non-commercial. See: http:// creativecommons.org/licenses/by-nc/4.0/

\section{REFERENCES}

1. Chareonthaitawee P, Gibbons RJ, Roberts RS, et al. The impact of time to thrombolytic treatment on outcome in patients with acute myocardial infarction. For the CORE investigators (Collaborative Organisation for RheothRx Evaluation). Heart 2000;84:142.

2. Sutton AG, Campbell PG, Graham R, et al. A randomized trial of rescue angioplasty versus a conservative approach for failed fibrinolysis in ST-segment elevation myocardial infarction: the Middlesbrough Early Revascularization to Limit INfarction (MERLIN) trial. J Am Coll Cardiol 2004;44:287.

3. Langer A, Krucoff MW, Klootwijk P, et al. Prognostic significance of ST segment shift early after resolution of ST elevation in patients with myocardial infarction treated with thrombolytic therapy: the GUSTO-I ST Segment Monitoring Substudy. J Am Coll Cardiol 1998:31:783.

4. The Global Use of Strategies to Open Occluded Coronary Arteries in Acute Coronary Syndromes (GUSTO Ilb) Angioplasty Substudy Investigators. A clinical trial comparing primary coronary angioplasty with tissue plasminogen activator for acute myocardial infarction. N Engl J Med 1997;336:1621.

5. Huynh T, Perron S, O'Loughlin J, et al. Comparison of primary percutaneous coronary intervention and fibrinolytic therapy in
ST-segment-elevation myocardial infarction: bayesian hierarchical meta-analyses of randomized controlled trials and observational studies. Circulation 2009;119:3101.

6. Gibson CM, Pride YB, Frederick PD, et al. Trends in reperfusion strategies, door-to-needle and door-to-balloon times, and in-hospital mortality among patients with ST-segment elevation myocardial infarction enrolled in The National Registry of Myocardial Infarction from 1990 to 2006. Am Heart J 2008;156:1035.

7. Morishima I, Sone T, Okumura K, et al. Angiographic no-reflow phenomenon as a predictor of adverse long-term outcome in patients treated with percutaneous transluminal coronary angioplasty for first acute myocardial infarction. J Am Coll Cardiol 2000;36:1202.

8. Fröbert O, Lagerqvist B, Olivecrona GK, et al. Thrombus aspiration during ST-segment elevation myocardial infarction. N Engl J Med 2013;369:1587-97.

9. Lagerqvist $\mathrm{B}$, Fröbert $\mathrm{O}$, Olivecrona $\mathrm{G}$, et al. Outcomes 1 year after thrombus aspiration for myocardial infarction. New Eng $\mathrm{J}$ Med 2014;371:1111-20.

10. Berger PB, Ellis SG, Holmes DR Jr, et al. Relationship between delay in performing direct coronary angioplasty and early clinical outcome in patients with acute myocardial infarction: results from the global use of strategies to open occluded arteries in Acute Coronary Syndromes (GUSTO-IIb) Trial. Circulation 1999;100:14.

11. Brodie BR, Hansen C, Stuckey TD, et al. Door-to-balloon time with primary percutaneous coronary intervention for acute myocardial infarction impacts late cardiac mortality in high-risk patients and patients presenting early after the onset of symptoms. J Am Coll Cardiol 2006;47:289.

12. Stone GW, Webb J, Cox DA, et al. Distal microcirculatory protection during percutaneous coronary intervention in acute ST-segment elevation myocardial infarction: a randomized controlled trial. JAMA 2005;293:1063. 